\title{
Role of metadherin in estrogen-regulated gene expression
}

\author{
YUJUN LI ${ }^{1}$, JESUS GONZALEZ BOSQUET ${ }^{1}$, SHUJIE YANG ${ }^{1}$, KRISTINA W. THIEL ${ }^{1}$, YUPING ZHANG ${ }^{1}$, \\ HAITAO LIU ${ }^{1}$, KIMBERLY K. LESLIE ${ }^{1,2}$ and XIANGBING MENG ${ }^{1,2}$ \\ ${ }^{1}$ Department of Obstetrics and Gynecology and ${ }^{2}$ Holden Comprehensive Cancer Center, \\ University of Iowa, Iowa City, IA 52242, USA
}

Received October 14, 2016; Accepted May 12, 2017

DOI: $10.3892 /$ ijmm.2017.3020

\begin{abstract}
The disruption of estrogen signaling is widely associated with the development of breast, endometrial and ovarian cancers. As a multifunctional mediator of carcinogenesis, metadherin (MTDH)/astrocyte elevated gene-1 (AEG-1) overexpression has been associated with numerous types of cancer, with reported roles in tumor initiation, proliferation, invasion, metastasis and chemoresistance. At the molecular level, MTDH has been shown to interact with proteins that drive tumorigenesis, including nuclear factor- $\kappa \mathrm{B}(\mathrm{NF}-\kappa \mathrm{B})$, promyelocytic leukaemia zinc finger (PLZF), BRCA2- and CDKN1A (p21Cip1/Waf-1/ mda-6)-interacting protein $\alpha(\mathrm{BCCIP} \alpha)$ and staphylococcal nuclease and tudor domain containing 1 (SND1). Through the analysis of the Cancer Genome Atlas (TCGA) datasets for estrogen receptor (ER)-positive endometrial and breast cancers, we found that over $25 \%$ of all gene expression correlated with MTDH. Using Affymetrix microarrays, we characterized the differences in gene expression between estrogen-treated parental and MTDH-deficient endometrial and breast cancer cells. We also explored a possible interaction between MTDH and ER by immunoprecipitation, and found that MTDH and ER associated in both breast and endometrial cancer cells in response to estrogen. Reciprocal co-immunoprecipitation analysis demonstrated that acute estrogen stimulation promoted the interaction of MTDH with ER in the nucleus. These data, to the best of our knowledge, provide the first evidence that MTDH and ER $\alpha$ interact in the nucleus with estrogen treatment to regulate gene expression.
\end{abstract}

\section{Introduction}

Metadherin (MTDH), also known as astrocyte elevated gene-1 (AEG-1) or LYRIC, is a protein encoded by the $M T D H$ gene. MTDH is frequently overexpressed in cancers of the

Correspondence to: Dr Xiangbing Meng, Department of Obstetrics and Gynecology, University of Iowa, 3234 MERF, 375 Newton Road, Iowa City, IA 52242, USA

E-mail: xiangbing-meng@uiowa.edu

Key words: metadherin, estrogen receptor, estrogen, endometrial cancer, breast cancer breast, prostate, ovary and endometrium, and is associated with poor clinical outcomes $(1,2)$. MTDH influences several oncogenic signaling pathways and transcription factors, such as the Ras, Myc, PI3K/AKT, nuclear factor $-\kappa \mathrm{B}(\mathrm{NF}-\kappa \mathrm{B})$, mitogen-activated protein kinase (MAPK) and Wnt pathways $(3,4)$, suggesting that MTDH plays an important role in disease development and progression.

MTDH is highly basic and contains a transmembrane domain and multiple nuclear localization signals, with reported localizations in the cell membrane, cytoplasm, nucleus, nucleolus and endoplasmic reticulum. Not surprisingly, distinct interaction partners have been reported for the various locales, allowing MTDH to contribute to a diverse range of intracellular events (4). For example, MTDH has been shown to interact with several proteins to drive tumorigenesis, including NF- $\kappa \mathrm{B}$, promyelocytic leukaemia zinc finger (PLZF), BRCA2- and CDKN1A (p21Cip1/Waf-1/mda-6)-interacting protein $\alpha(\mathrm{BCCIP} \alpha)$ and staphylococcal nuclease and tudor domain containing 1 (SND1) (5-8). MTDH also interacts with the steroid hormone receptors $\operatorname{RXRs}(\operatorname{RXR} \alpha, \beta, \gamma)$ to negatively regulate $\mathrm{RXR}$ function and $\mathrm{RXR}$-mediated transcriptional regulation (9). Recently, some reports have suggested that MTDH overexpression inversely correlates with the estrogen receptor (ER) status, and $\mathrm{MTDH}$ may induce resistance to tamoxifen in ER $\alpha$-positive breast cancer cells (10-12).

In this study, we examined the potential association between MTDH and ER. Estrogens control multiple cellular functions via estrogen receptors (ER- $\alpha$ and ER- $\beta$ ), modular proteins that belong to the steroid/nuclear receptor family of intracellular homeostatic regulators. Ligand binding in the cytosol results in conformation changes that promote receptor translocation into the nucleus, thereby promoting gene expression or repression through interactions with chromatin and transcriptional co-regulatory complexes. In this study, we found that the expression of a number of genes correlated with MTDH expression in ER positive cancers from the Cancer Genome Atlas (TCGA) dataset, and we also found a transient interaction of MTDH with $\mathrm{ER} \alpha$ following estrogen treatment.

\section{Materials and methods}

The Cancer Genome Atlas (TCGA) data analysis. Gene expression data from the RNA sequencing (RNA-seq) experiments were downloaded from the TCGA (https://portal.gdc. cancer.gov/) data portal for breast and endometrial cancer. For 
RNA-seq only level 3 data was available. Data at level 3 are already preprocessed, normalized and $\log 2$-transformed (for more details see https://cancergenome.nih.gov/abouttcga/aboutdata/datalevelstypes). In addition, metadata and clinical data were extracted and downloaded to identify clinical parameters, such as endometrial histological types (serous vs. endometrioid), and molecular characteristics, such as ER-positive vs. ER-negative breast cancer. Table I shows the patients included in the analysis.

To abstract unique gene expression data we used the Biometric Research Branch (BRB) ArrayTools (version 2.13.2 for X64 systems) software suite. BRB-ArrayTools was developed by Dr Richard Simon and colleagues, and is an integrated package for visualization and statistical analysis utilizing Excel (Microsoft, Redmond, WA, USA) as the front end, with tools developed in the R statistical system. MTDH expression was correlated with the expression of all other genes in breast and endometrial RNA-seq experiments using rank-based Spearman correlation to allow for non-linear relationships between several gene expression values. To control the false discovery rate (FDR) we used the q-value for statistical significance (qFDR) and Bonferroni correction for multiple comparisons. A significant correlation was considered a correlation with a $\mathrm{p}$-value $<10^{-6}$ after controlling for multiple comparisons.

Cell culture and generation of MTDH-deficient cells. The human endometrial cancer cell line, ECC1 (American Type Culture Collection; ATCC, Manassas, VA, USA), was maintained in RPMI-1640 medium supplemented with $10 \%$ fetal bovine serum (FBS) and 1\% penicillin/streptomycin, MCF-7 cells (ATCC) were maintained in Dulbecco's modified Eagle's medium (DMEM) medium supplemented with 10\% FBS and $1 \%$ penicillin/streptomycin (all from Gibco-BRL, Carlsbad, CA, USA); all cells were incubated at $37^{\circ} \mathrm{C}$ in a humidified atmosphere of $5 \% \mathrm{CO}_{2}$. Genomic deletions of $\mathrm{MTDH}$ were created in the ECC1 cells using 3 pairs of chimeric single guide RNAs (sgRNAs) as previously described (13). Briefly, sgRNA oligos were synthesized and cloned into the lentiCRISPR transfer plasmid for virus production. Viral vectors were produced in 293T cells (ATCC), followed by infection of ECC1 cells as described. MTDH-deficient ECC1 cells were selected with $5 \mu \mathrm{g} /$ $\mathrm{ml}$ puromycin after $48 \mathrm{~h}$ of transduction. MTDH-deficient ECC1 cells were grown at low density for 2 weeks to select clones. MTDH expression was determined by western blot analysis to identify clones with no or low MTDH protein expression.

Microarray assay and data analysis. ECC1 and MTDH-deficient ECC1 cells were incubated in RPMI-1640 supplemented with $5 \%$ charcoal stripped FBS for $24 \mathrm{~h}$ then treated with $10 \mathrm{nM}$ estrogen (Sigma-Aldrich, St. Louis, MO, USA) or treated with ethanol as a control for $24 \mathrm{~h}$. Total RNA was isolated using the Qiagen RNeasy mini kit (Qiagen, Valencia, CA, USA). RNA sample preparation for hybridization and the subsequent hybridization to the Illumina beadchips were performed at the University of Iowa IIHG Genomics Division using the manufacturer's recommended protocols. Briefly, $100 \mathrm{ng}$ total RNA were converted to amplified biotin-aRNA using the Epicentre TargetAmp-Nano Labeling kit for Illumina Expression BeadChip (Illumina, Inc., San Diego, CA, USA). The amplified
Biotin-aRNA product was purified through a Qiagen RNeasy MinElute Cleanup column (Qiagen) according to modifications from Epicentre (Madison, WI, USA). Subsequently, $750 \mathrm{ng}$ of this product were mixed with Illumina hybridization buffer, placed onto Illumina-Human H12 v4 BeadChips (part no. BD-103-0204), and incubated at $58^{\circ} \mathrm{C}$ for $17 \mathrm{~h}$, with rocking, in an Illumina hybridization oven. Following hybridization, the arrays were washed, blocked and then stained with streptavidin-Cy3 (Amersham/GE Healthcare, Piscataway, NJ, USA) according to the Illumina Whole-Genome Gene Expression Direct Hybridization Assay protocol. Beadchips were scanned with the Illumina iScan system (ID \#N0534) and data were collected using the GenomeStudio software v2011.1. Microarray data have been deposited at GEO (www.ncbi.nlm. nih.gov/geo) under the accession no. GSE78182. The microarray data were analyzed for data summarization, normalization and quality control by using BRB ArrayTools (version 2.13.2 for X64 systems) software suite. To select the differentially expressed genes, we used threshold values of $\geq 2$ and $\leq$-2-fold-change and a Benjamini-Hochberg corrected $\mathrm{p}$-value of 0.001 . The data were $\log 2$-transformed and median centred by genes using the Adjust Data function of Cluster 3.0 software, and then further analyzed with hierarchical clustering with average linkage. The microarray experiment was performed using biological triplicates.

Immunoprecipitation (IP) and western blot analysis. The ECC1 cells were incubated in RPMI-1640 and the MCF-7 cells were incubated in DMEM supplemented with 5\% charcoal stripped FBS for $24 \mathrm{~h}$ then treated with $10 \mathrm{nM}$ estrogen for $0,1,2$ and $4 \mathrm{~h}$. For IP, the cells were lysed in $400 \mu \mathrm{l}$ of NP-40 buffer and $5 \%$ of the lysates were saved as input. BCA protein assay was used to determine the protein concentrations of each lysate. A total of $1 \mathrm{mg}$ of the protein lysates were incubated with $1 \mu \mathrm{g}$ primary antibodies followed by incubation with protein $A / G$ agarose beads (Santa Cruz Biotechnology, Inc., Santa Cruz, CA, USA), which were then washed 4 times with NP-40 buffer. Immuno-complexes were boiled in $2 \mathrm{X}$ Laemmli sample buffer for $5 \mathrm{~min}$ before they were subjected to sodium dodecyl sulfate-polyacrylamide gel electrophoresis (SDS-PAGE), transferred onto nitrocellulose membranes and immunoblotted with MTDH antibody (cat. no. 40-6500; Invitrogen) or ER $\alpha$ antibody (cat. no. sc-543; Santa Cruz Biotechnology, Inc.). The procedures for cytoplasmic and nuclear extraction were carried out according the manufacture'rs instructions (Active Motif, Carlsbad, CA, USA), and the fractions were then immunoprecipitated with ER $\alpha$ antibody, followed by immunoblotting with $\mathrm{ER} \alpha$ or MTDH antibodies.

Immunostaining. Breast cancer and endometrial cancer specimens obtained from the Tissue Procurement Core were subjected to immunohistochemical staining for either ER or MTDH as previously described (13).

$R T-q P C R$. Four significant genes from microarray data were randomly selected and validated by RT-qPCR analysis in the ECC1 cells. Total RNA was isolated using the Qiagen RNeasy mini kit (Qiagen), and reverse transcription with SuperScript ${ }^{\circledR}$ III First-Strand Synthesis system (Invitrogen). SYBR-Green-based real-time PCR (SYBR-Green I; ABI, Warrington, UK) was used to quantitatively detect the expres- 
Table I. Number of genes correlated with MTDH expression $\left(\mathrm{p}<10^{-6}\right)$.

\begin{tabular}{lcccc}
\hline & \multicolumn{2}{c}{$\begin{array}{c}\text { Endometrial cancer } \\
(18,023 \text { genes })\end{array}$} & & $\begin{array}{c}\text { Breast cancer } \\
(17,660 \text { genes })\end{array}$ \\
\cline { 2 - 5 } & $\begin{array}{c}\text { ER-Pos (endometrioid) } \\
(\mathrm{N}=142)\end{array}$ & $\begin{array}{c}\text { ER-Neg (serous) } \\
(\mathrm{N}=38)\end{array}$ & $\begin{array}{c}\text { ER-Pos } \\
(\mathrm{N}=705)\end{array}$ & $\begin{array}{c}\text { ER-Neg } \\
(\mathrm{N}=207)\end{array}$ \\
\hline Significant ${ }^{2}$ with MTDH & 4479 & 88 & 4,451 & 332 \\
$\%$ Correlation & $24.9 \%$ & $0.5 \%$ & $25.2 \%$ & $1.9 \%$ \\
\hline
\end{tabular}

MTDH, metadherin; ER, estrogen receptor; Pos, positive; Neg, negative.

sion of Phosphatidic Acid Phosphatase Type 2B (PPAP2B), Plexin Domain Containing 2 (PLXDC2), Transmembrane And Tetratricopeptide Repeat Containing 1 (TMTC1), Protease, Serine 23 (PRSS23) and 18S RNA. The fold variation in the RNA samples was calculated using the $2^{-\Delta \Delta c t}$ method following normalization with $18 \mathrm{~S}$. The primer sequences were as follows: PPAP2B forward, 5'-TGA GAG CAT CAA GTA CCC ACT-3' and reverse, 5'-ACG TAG GGG TTC TGA ATC GTC-3'; PLXDC2 forward, 5'-TTC TCA AGG CGG TAG ACA CGA-3' and reverse, 5'-CGA TCT GAG TGT TAT TGT CCT GC-3'; TMTC1 forward, 5'-ACG GTG TCT CCC TTC TTC TTG-3' and reverse, 5'-ATT GCT CGA CTT GTC TTG CTT-3'; PRSS23 forward, 5'-CAG TGT CAT AAG GGA ACT CCA C-3' and reverse, 5'-CCT GAG TCT CGG TGT TGG G-3'; 18S forward, 5'-AAC TTT CGA TGG TAG TCG CCG-3' and reverse, 5'-CCT TGG ATG TGG TAG CCG TTT-3'.

Online tools. Gene ontology (GO) analyses were based on the online tool PANTHER (http://pantherdb.org/).

\section{Results}

The expression of a number of genes correlates with MTDH expression in ER-positive endometrial cancer and breast cancer. Using the Cancer Genome Atlas (TCGA) dataset, with a cut-off set at p-value $<10^{-6}$ between endometrial cancer and serous endometrial cancer, or between ER-positive and ER-negative breast cancer, we found that the expression of $24.9 \%$ of the genes correlated with MTDH expression in endometrial cancer, and the expression of $25.2 \%$ of the genes correlated with MTDH expression in ER-positive breast cancer (Table I). By contrast, only approximately 0.5 or $1.9 \%$ of the genes correlated with MTDH expression in serous endometrial cancer and ER-negative breast cancer, respectively. These results indicate that MTDH may be associated with ER or estrogen-regulated gene expression.

Microarray analysis of estrogen-treated ECC1 cells and MTDH-deficient ECCl cells. To further examine the association between MTDH and ER, genomic deletions of MTDH were created in ECC1 cells (MTDH-deficient ECC1 cells). The expression levels of MTDH and ER were detected by western blot analysis in these cells (Fig. 1A), and the differences in gene expression between estrogen-treated parental ECC1 cells and MTDH-deficient ECC1 cells were then investigated using microarray assay. By using threshold values of $\mathrm{p}<0.001$ and fold-change $\geq 2$ or $\leq-2$, the ECC1 cells had 46 and the MTDHdeficient ECC1 cells had 42 significantly altered genes induced by estrogen treatment (Fig. 1B). Of note, 27 genes were induced by estrogen in both parental the ECC1 and MTDH-deficient ECC1 cells (Table II). These genes are estrogen induced and not related with MTDH status, and thus these genes may be associated with the function of estrogen receptors independent of MTDH. There were 19 estrogen-regulated genes that were dependent on MTDH expression (Table III). Molecular network analysis did not find that these differentially expressed genes had direct interactions with MTDH (data not shown). We also found 15 genes significantly regulated by estrogen treatment in the MTDH-deficient ECC1 cells (Table IV).

We randomly selected 2 upregulated and 2 downregulated genes and validated the expression changes in the ECC1 cells by RT-qPCR. The fold-change of these genes was similar with the microarray data (Fig. 1C). The differential gene expression induced by estrogen in the ECC1 and MTDH-deficient ECC1 cells was represented graphically in a heatmap (Fig. 1D and F).

Inputting 46 differentially expressed genes induced by estrogen in the ECC1 cells into the online tool PANTHER (http://pantherdb.org/) identified 47 terms belonging to molecular functions (Fig. 1E) and 90 terms belonging to biological processes (data not shown). Inputting 42 differentially expressed genes induced by estrogen in the MTDH-deficient ECC1 cells into the online tool PANTHER identified 46 terms belonging to molecular functions (Fig. 1G) and 97 terms belonging to biological processes (data not shown). The most representative molecular functions were binding and catalytic. On the other hand, the most significant biological processes involving the ER interacting proteins identified are represented by metabolic processes and cellular process (data not shown).

ER and MTDH interact in the nucleus. In order to define the involvement of MTDH in ER or estrogen-regulated gene expression, the ECC1 endometrial cancer cells were treated with $10 \mathrm{nM}$ estrogen for $0,1,2$ and $4 \mathrm{~h}$, and the interaction of MTDH and ER $\alpha$ was queried using IP assays. The IP results revealed that MTDH and ER $\alpha$ can 'pull down' each other following estrogen treatment for $2 \mathrm{~h}$ (Fig. 2A). We acquired similar results in the MCF-7 cells (Fig. 2B).

We then wished to determine where MTDH and ER $\alpha$ interact in the cell. Cytoplasmic and nuclear fractions of ECC1 cells treated with $10 \mathrm{nM}$ estrogen were immunoprecipitated with anti-ER $\alpha$ antibody, followed by immunoblotting with ER $\alpha$ or MTDH antibody. The results indicated that the interaction of 


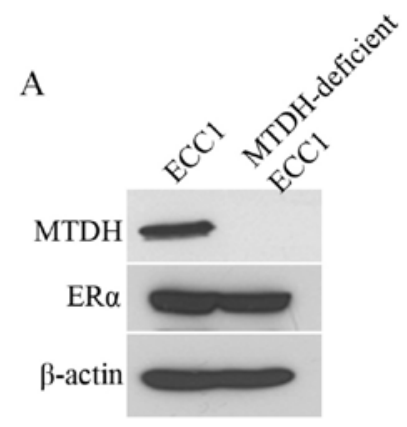

B

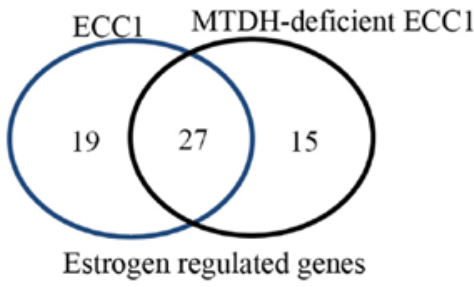

D

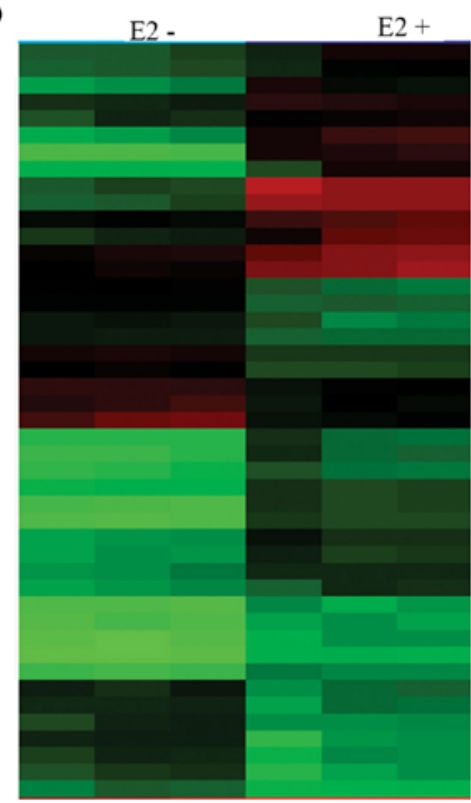

E
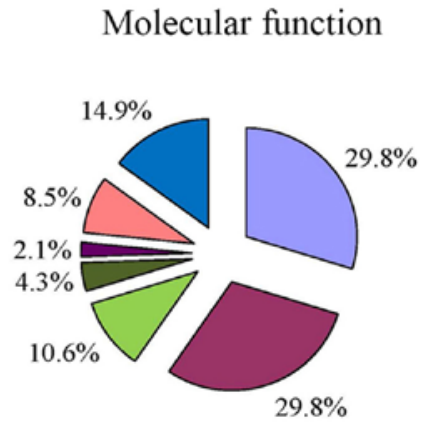

$\mathrm{F}$

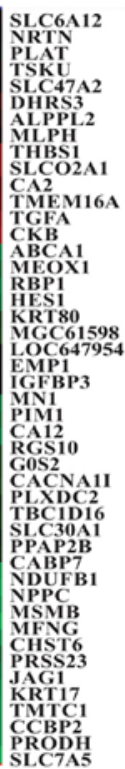

C

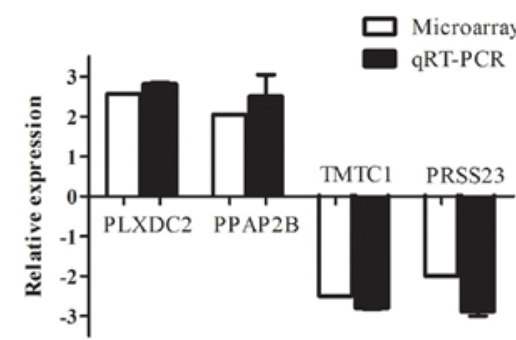

MTDH-deficient ECCl

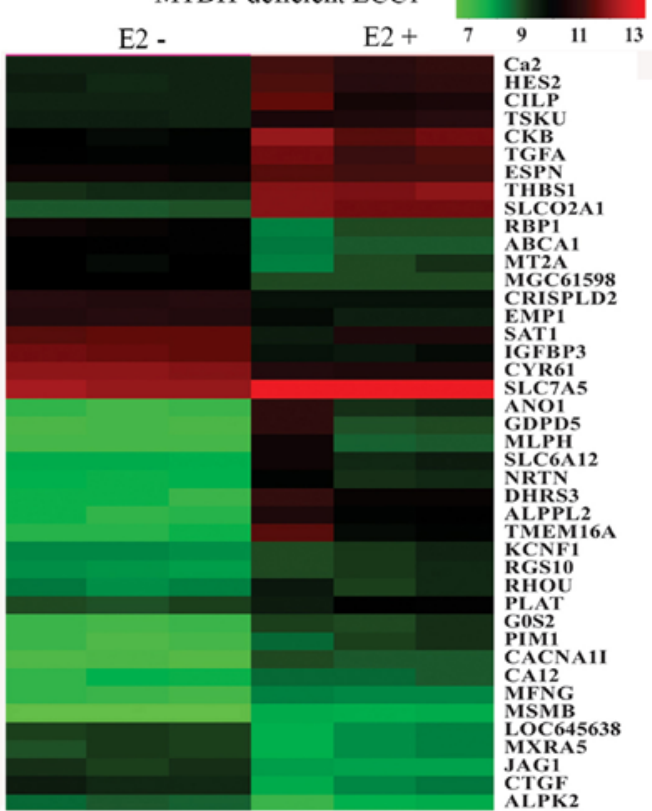

G

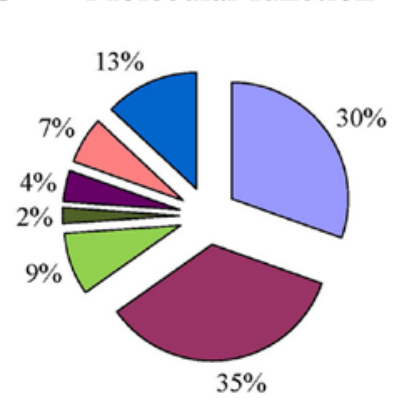

$\square$ binding

a catalytic activity

$\square$ enzyme regulator activity

๘ nucleic acid binding

transcription factor activity

- receptor activity

$\square$ structural molecule activity

घ transporter activity

Figure 1. Microarray data analysis of estrogen-treated control and metadherin (MTDH)-deficient ECC1 cells. (A) The expression of MTDH and estrogen receptor $\alpha(E R \alpha)$ in ECC1 cells and MTDH-deficient ECC1 cells. (B) Venn diagram of differentially expressed genes induced by estrogen between ECC1 and MTDH-deficient ECC1 cells. (C) Two upregulated genes and two downregulated genes were amplified from estrogen-treated ECC1 cells and control ECC1 cells, and the fold-change compared with microarray data. (D and F) Heatmap of estrogen induced differently expressed genes in ECC1 cells (D) or in MTDHdeficient ECC1 cells (F). (E and G) GO analysis of estrogen-induced differently expressed genes in ECC1 cells (E) or MTDH-deficient ECC1 cells associated with molecular functions $(\mathrm{G})$.

MTDH and ER $\alpha$ occurs in the nucleus (Fig. 2C). Furthermore, we detected the co-localization of MTDH and ER by immunostaining in a subset of cells in ER-positive breast cancer and endometrial cancer tissues (Fig. 3).

\section{Discussion}

Cancer progression is a multifactorial process that includes alterations in proliferation, cell death, apoptosis, genetic instability, and changes in the expression and activity of tumor suppressor genes, oncogenes, growth factors and cell adhesion molecules (14-16). The overexpression of MTDH is frequently observed in most human cancers, and several studies have suggested that MTDH may play a pivotal role in the pathogenesis, invasion, metastasis, angiogenesis, progression and regulation of apoptosis. Estrogen receptors are overexpressed in approximately $70 \%$ of breast and endometrium tumors. Similar to MTDH, estrogen signaling through ER promotes cell proliferation and survival and inhibits apoptosis, thereby promoting tumorigenesis. In this study, we found that the 
Table II. MTDH-independent gene expression regulated by estrogen in ECC1 cells (MTDHWT) and MTDH-deficient ECC1 (MTDH KO) cells ( $\mathrm{p}<0.001$ and fold-change $\geq 2$ or $\leq-2$ ).

\begin{tabular}{|c|c|c|c|c|}
\hline \multirow[b]{2}{*}{ UniqueID } & \multirow[b]{2}{*}{ Name } & \multirow[b]{2}{*}{$\begin{array}{l}\text { Map } \\
\text { Location }\end{array}$} & \multicolumn{2}{|c|}{$\begin{array}{l}\text { Fold-change } \\
\text { (treat/no-treat) }\end{array}$} \\
\hline & & & $\begin{array}{l}\text { MTDH } \\
\text { WT }\end{array}$ & $\begin{array}{l}\text { MTDH } \\
\text { KO }\end{array}$ \\
\hline ALPPL2 & Alkaline phosphatase, placental-like 2 (ALPPL2) & $2 \mathrm{q} 37.1 \mathrm{c}$ & 12.06 & 7.50 \\
\hline THBS1 & Thrombospondin 1 (THBS1) & $15 \mathrm{q} 14 \mathrm{~d}$ & 9.50 & 6.45 \\
\hline SLCO2A1 & Solute carrier organic anion transporter family, member $2 \mathrm{~A} 1$ (SLCO2A1) & $3 \mathrm{q} 22.1 \mathrm{e}$ & 9.45 & 8.33 \\
\hline DHRS3 & Dehydrogenase/reductase (SDR family) member 3 (DHRS3) & $\begin{array}{l}1 \mathrm{p} 36.22 \mathrm{a}- \\
\mathrm{p} 36.21 \mathrm{~d}\end{array}$ & 7.92 & 9.38 \\
\hline MLPH & Melanophilin (MLPH), transcript variant 2 & $2 q 37.3 b$ & 5.19 & 4.60 \\
\hline CACNA1I & $\begin{array}{l}\text { Calcium channel, voltage-dependent, } \mathrm{T} \text { type, alpha } 1 \mathrm{I} \text { subunit (CACNA1I), } \\
\text { transcript variant } 1\end{array}$ & $22 q 13.1 d$ & 4.18 & 3.81 \\
\hline G0S2 & G0/G1 switch 2 (G0S2) & $1 \mathrm{q} 32.2 \mathrm{~b}$ & 4.04 & 3.81 \\
\hline PLAT & Plasminogen activator, tissue (PLAT), transcript variant 1 & $8 \mathrm{p} 11.21 \mathrm{a}$ & 3.66 & 2.02 \\
\hline TMEM16A & Transmembrane protein 16A (TMEM16A) & $\begin{array}{l}11 \mathrm{q} 13.3 \mathrm{~b}- \\
\mathrm{q} 13.3 \mathrm{c}\end{array}$ & 3.60 & 8.52 \\
\hline CKB & Creatine kinase, brain (CKB) & $14 q 32.33 \mathrm{a}$ & 3.41 & 3.68 \\
\hline PIM1 & Pim-1 oncogene (PIM1) & $6 \mathrm{p} 21.2 \mathrm{c}$ & 3.01 & 3.59 \\
\hline $\mathrm{CA} 2$ & Carbonic anhydrase II (CA2) & $8 \mathrm{q} 21.2 \mathrm{~b}$ & 2.82 & 3.29 \\
\hline RGS10 & Regulator of G-protein signaling 10 (RGS10), transcript variant 1 & $10 \mathrm{q} 26.11 \mathrm{~d}$ & 2.80 & 2.16 \\
\hline TSKU & Tsukushin (TSKU), mRNA. & $11 \mathrm{q} 13.5 \mathrm{c}$ & 2.64 & 2.42 \\
\hline TGFA & Transforming growth factor alpha (TGFA), transcript variant 2 & $2 \mathrm{p} 13.3 \mathrm{c}$ & 2.52 & 2.86 \\
\hline SLC6A12 & $\begin{array}{l}\text { Solute carrier family } 6 \text { (neurotransmitter transporter, betaine/GABA), } \\
\text { member } 12 \text { (SLC6A12) }\end{array}$ & $12 \mathrm{p} 13.33 \mathrm{~d}$ & 2.41 & 4.11 \\
\hline CA12 & Carbonic anhydrase XII (CA12), transcript variant 1 & $15 q 22.2 b$ & 2.30 & 2.28 \\
\hline MFNG & MFNG O-fucosylpeptide 3-beta-N-acetylglucosaminyltransferase (MFNG) & $22 q 13.1 \mathrm{a}$ & 2.15 & 2.10 \\
\hline SLC7A5 & $\begin{array}{l}\text { Solute carrier family } 7 \text { (cationic amino acid transporter, } \mathrm{y}^{+} \text {system), } \\
\text { member } 5 \text { (SLC7A5) }\end{array}$ & $16 \mathrm{q} 24.2 \mathrm{~b}$ & 2.07 & 2.22 \\
\hline MSMB & Microseminoprotein beta (MSMB), transcript variant PSP57 & $10 q 11.23 \mathrm{~b}$ & 2.03 & 2.08 \\
\hline NRTN & Neurturin (NRTN) & $19 \mathrm{p} 13.3 \mathrm{~b}$ & 2.03 & 3.89 \\
\hline JAG1 & Jagged 1 (Alagille syndrome) (JAG1) & $20 \mathrm{p} 12.2 \mathrm{a}$ & -2.18 & -2.41 \\
\hline RBP1 & Retinol binding protein 1 , cellular (RBP1) & $3 q 23 a$ & -2.19 & -2.98 \\
\hline MGC61598 & $\begin{array}{l}\text { PREDICTED: Homo sapiens similar to ankyrin-repeat } \\
\text { protein Nrarp (MGC61598) }\end{array}$ & $9 q 34.3 f$ & -2.22 & -2.16 \\
\hline EMP1 & Epithelial membrane protein 1 (EMP1) & $12 \mathrm{p} 13.1 \mathrm{~b}$ & -2.23 & -2.29 \\
\hline ABCA1 & ATP-binding cassette, sub-family A (ABC1), member 1 (ABCA1) & $9 q 31.1 d$ & -2.83 & -2.81 \\
\hline IGFBP3 & Insulin-like growth factor binding protein 3 (IGFBP3), transcript variant 2 & $7 \mathrm{p} 13 \mathrm{~b}$ & -3.32 & -3.82 \\
\hline
\end{tabular}

MTDH, metadherin; treat/no treat, treatment or not with estrogen.

expression of many genes correlated with the expression of MTDH in ER-positive cancers using TCGA data, and we defined an interaction between MTDH and ER by co-IP. Our data suggest that the interaction is dependent on estrogen and is a short-term process.

ERs are involved in pathological processes, including breast cancer, endometrial cancer, osteoporosis, hypertension and coronary artery diseases (17-19). One of the main risk factors for endometrial cancer is high levels of estrogen (20). In this study, we used the human endometrial cancer cell line, ECC1, and MTDH-deficient ECC1 cells, treated with or without estrogen, and assessed the gene expression profiles by microarray assay. Our results demonstrated that most estrogen-induced genes were common in the ECC1 cells and MTDH-deficient ECC1 cells, indicating that approximately $60 \%$ of the genes induced by estrogen were unrelated with the MTDH status, and that MTDH may only participate in parts of ER function.

Previous studies have indicated that MTDH interacts with and activates the transcription factor, $\mathrm{NF}-\kappa \mathrm{B}$, to regulate gene expression $(3,8,21)$. In this study, we found that MTDH transiently interacted with ER in the nucleus of cancer cells, the interaction is dependent on estrogen and it is a short-term 
Table III. List of differently expressed MTDH-dependent genes regulated by estrogen in ECC1 cells ( $p<0.001$ and fold-change $\geq 2$ or $\leq-2)$.

\begin{tabular}{lcll}
\hline UniqueID & $\begin{array}{c}\text { Fold-change } \\
\text { (treat/no-treat) }\end{array}$ & \multicolumn{1}{c}{ Name } & MapLocation \\
\hline PLXDC2 & 2.57 & Plexin domain containing 2 (PLXDC2) & 10p12.32a- \\
p12.31c \\
MN1 & 2.54 & Meningioma (disrupted in balanced translocation) 1 (MN1) & 22q12.1b \\
NPPC & 2.36 & Natriuretic peptide precursor C (NPPC) & 2q37.1b \\
TBC1D16 & 2.27 & TBC1 domain family, member 16 (TBC1D16), mRNA. & $17 \mathrm{q} 25.3 \mathrm{~d}$ \\
SLC30A1 & 2.21 & Solute carrier family 30 (zinc transporter), member 1 (SLC30A1) & $1 \mathrm{q} 32.3 \mathrm{a}$ \\
SLC47A2 & 2.17 & Solute carrier family 47, member 2 (SLC47A2), transcript variant 1 & $17 \mathrm{p} 11.2 \mathrm{~d}$ \\
CABP7 & 2.06 & Calcium binding protein 7 (CABP7) & $22 \mathrm{q} 12.2 \mathrm{a}$ \\
PPAP2B & 2.05 & Phosphatidic acid phosphatase type 2B (PPAP2B), transcript variant 2 & $1 \mathrm{p} 32.2 \mathrm{c}$ \\
NDUFB1 & 2.03 & NADH dehydrogenase (ubiquinone) 13 subcomplex, 1, 7 kDa (NDUFB1) & $14 \mathrm{q} 32.12 \mathrm{~b}$ \\
LOC647954 & -2.00 & PREDICTED: Homo sapiens misc_RNA (LOC647954) & $5 \mathrm{q} 23.2 \mathrm{~b}$ \\
PRODH & -2.00 & Proline dehydrogenase (oxidase) 1 (PRODH) & $22 \mathrm{q} 11.21 \mathrm{~b}$ \\
CHST6 & -2.00 & Carbohydrate (N-acetylglucosamine 6-O) sulfotransferase 6 (CHST6) & $16 \mathrm{q} 23.1 \mathrm{a}$ \\
HES1 & -2.01 & Hairy and enhancer of split 1, (Drosophila) (HES1) & $3 \mathrm{q} 29 \mathrm{c}$ \\
PRSS23 & -2.01 & Protease, serine, 23 (PRSS23) & $11 \mathrm{q} 14.2 \mathrm{a}$ \\
MEOX1 & -2.34 & Mesenchyme homeobox 1 (MEOX1), transcript variant 3 & $17 \mathrm{q} 21.31 \mathrm{~b}$ \\
CCBP2 & -2.44 & Chemokine binding protein 2 (CCBP2) & $3 \mathrm{p} 22.1 \mathrm{a}$ \\
TMTC1 & -2.49 & Transmembrane and tetratricopeptide repeat containing 1 (TMTC1) & $12 \mathrm{p} 11.22 \mathrm{a}$ \\
KRT80 & -2.58 & Keratin 80 (KRT80), transcript variant 1 & $12 \mathrm{q} 13.13 \mathrm{~d}$ \\
KRT17 & -3.02 & Keratin 17 (KRT17) & $17 \mathrm{q} 21.2 \mathrm{~b}$ \\
\hline
\end{tabular}

MTDH, metadherin; treat/no treat, treatment or not with estrogen.

Table IV. MTDH negatively regulated gene expression regulated by estrogen in MTDH-deficient ECC1 cells $(\mathrm{p}<0.001$ and fold-change $\geq 2$ or $\leq-2$ ).

\begin{tabular}{lcll}
\hline UniqueID & $\begin{array}{c}\text { Fold-change } \\
\text { (treat/no-treat) }\end{array}$ & \multicolumn{1}{c}{ Name } & MapLocation \\
\hline ANO1 & 6.46 & Anoctamin 1, calcium activated chloride channel (ANO1), transcript variant 1 & $\begin{array}{l}\text { 11q13.3b- } \\
\text { q13.3c }\end{array}$ \\
GDPD5 & 5.85 & Glycerophosphodiester phosphodiesterase domain containing 5 (GDPD5) & 11q13.4c- \\
& & & q13.5a \\
HES2 & 3.24 & Hairy and enhancer of split 2 (Drosophila) (HES2) & 1p36.31a \\
CILP & 2.90 & Cartilage intermediate layer protein, nucleotide pyrophosphohydrolase (CILP) & 15q22.31b \\
RHOU & 2.07 & Ras homolog gene family, member U (RHOU) & 1q42.13c \\
KCNF1 & 2.02 & Potassium voltage-gated channel, subfamily F, member 1 (KCNF1) & 2p25.1c \\
ESPN & 2.01 & Espin (ESPN) & 1p36.31a \\
MXRA5 & -2.00 & Matrix-remodelling associated 5 (MXRA5) & Xp22.33b \\
CRISPLD2 & -2.07 & Cysteine-rich secretory protein LCCL domain containing 2 (CRISPLD2) & $16 \mathrm{q} 24.1 \mathrm{a}$ \\
LOC645638 & -2.08 & PREDICTED: Homo sapiens misc_RNA (LOC645638), miscRNA & $17 \mathrm{q} 23.1 \mathrm{a}$ \\
MT2A & -2.21 & Metallothionein 2A (MT2A) & $16 \mathrm{q} 13 \mathrm{~b}$ \\
ALPK2 & -2.22 & Alpha-kinase 2 (ALPK2) & $18 \mathrm{q} 21.31 \mathrm{~b}-$ \\
& & & q21.32a \\
SAT1 & -2.44 & Spermidine/spermine N1-acetyltransferase 1 (SAT1) & Xp22.11a \\
CTGF & -2.45 & Connective tissue growth factor (CTGF) & 6q23.2b \\
CYR61 & -2.50 & Cysteine-rich, angiogenic inducer, 61 (CYR61) & 1p22.3e \\
\hline
\end{tabular}

MTDH, metadherin; treat/no treat, treatment or not with estrogen. 
A

WCE

IP: MTDH

IP: $\mathrm{ER} \alpha$

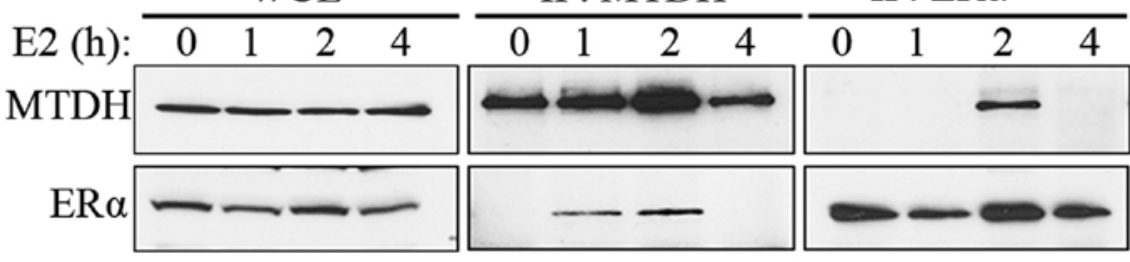

B

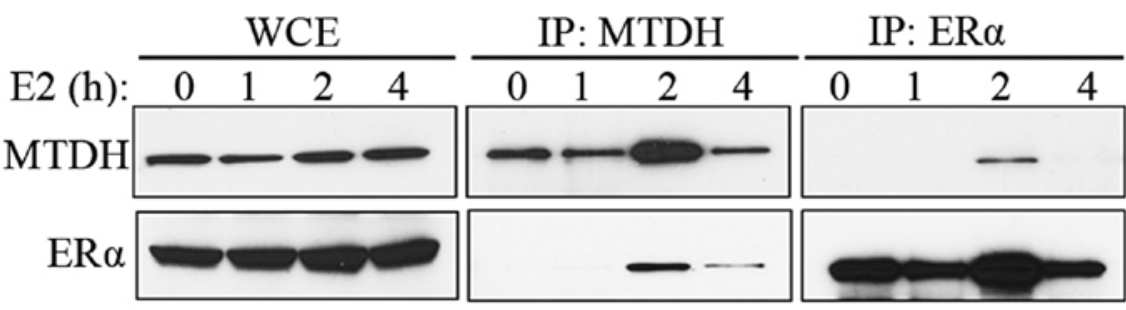

$\mathrm{C}$

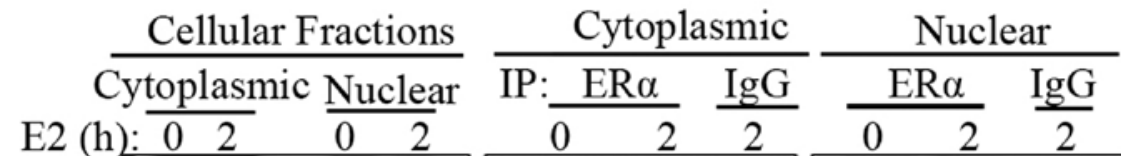

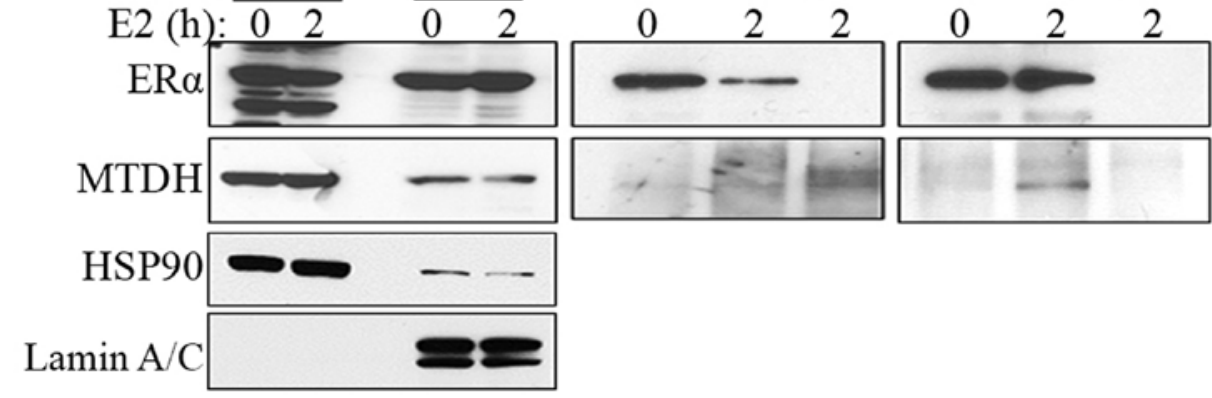

Figure 2. The interaction of estrogen receptor (ER) and metadherin (MTDH) in endometrial cancer and breast cancer cell lines. (A) Reciprocal co-IP analysis of the interaction between MTDH and ER in ECC1 cells. Cell lysates of ECC1 cells treated with $10 \mathrm{nM}$ estrogen were immunoprecipitated with anti-MTDH or anti-ER $\alpha$ antibody. (B) Reciprocal co-IP analysis of the interaction between MTDH and ER in MCF-7 cells. Cell lysates of MCF-7 cells treated with $10 \mathrm{nM}$ estrogen were immunoprecipitated with anti-MTDH or anti-ER antibody. (C) Reciprocal co-IP analysis of the fractions in ECC1 cells. Cytoplasmic and nuclear fractions of ECC1 cells treated with $10 \mathrm{nM}$ estrogen were immunoprecipitated with anti-ER $\alpha$ antibody.


Endometrial cancer

Figure 3. Co-localization of estrogen receptor (ER) and metadherin (MTDH) in breast cancer (A) and endometrial cancer (B) tissues. Co-localization was analyzed by dual immunostaining with anti-MTDH and anti-ER antibodies. Cells with co-localized MTDH and ER in the nucleus are indicated by arrows.

process. Based on this time course, the biological function of this transient interaction may relate to the transcriptional activity of ER $\alpha$. Indeed, the long-standing dogma in the hormone receptor field is that ligand binding and receptor- mediated transcriptional activation are highly transient events. Microarray data indicated that MTDH had some effect on estrogen-regulated genes, and they jointly control the expression of a subset of ER-regulated genes. MTDH thus may 
play an important role early in ER-mediated transcriptional regulation. Further studies on understanding the interaction of MTDH with ER and the related genes may provide new insight into the function of MTDH and ER in tumors.

\section{Acknowledgements}

This study was supported by National Institutes of Health (NIH) Grants R01CA184101 (to X.M. and K.K.L.) and R01CA99908 (to K.K.L.) and by the Department of Obstetrics and Gynecology Research Development Fund (to K.K.L.). Microarray data presented herein were obtained at the Genomics Division of the Iowa Institute of Human Genetics which is supported, in part, by the University of Iowa Carver College of Medicine and the Holden Comprehensive Cancer Center and its National Cancer Institute Award P30CA086862. It should be noted that K.W.T. is a co-owner of Immortagen, Inc.

\section{References}

1. Wang Z, Wei YB, Gao YL, Yan B, Yang JR and Guo Q: Metadherin in prostate, bladder, and kidney cancer: A systematic review. Mol Clin Oncol 2: 1139-1144, 2014

2. Song H, Li C, Lu R, Zhang Y and Geng J: Expression of astrocyte elevated gene-1: A novel marker of the pathogenesis, progression, and poor prognosis for endometrial cancer. Int J Gynecol Cancer 20: 1188-1196, 2010.

3. Emdad L, Sarkar D, Su ZZ, Randolph A, Boukerche H, Valerie K and Fisher PB: Activation of the nuclear factor kappaB pathway by astrocyte elevated gene-1: Implications for tumor progression and metastasis. Cancer Res 66: 1509-1516, 2006.

4. Yoo BK, Emdad L, Lee SG, Su ZZ, Santhekadur P, Chen D, Gredler R, Fisher PB and Sarkar D: Astrocyte elevated gene-1 (AEG-1): A multifunctional regulator of normal and abnormal physiology. Pharmacol Ther 130: 1-8, 2011.

5. Wan L, Lu X, Yuan S, Wei Y, Guo F, Shen M, Yuan M, Chakrabarti R, Hua Y, Smith HA, et al: MTDH-SND1 interaction is crucial for expansion and activity of tumor-initiating cells in diverse oncogene- and carcinogen-induced mammary tumors. Cancer Cell 26: 92-105, 2014

6. Ash SC, Yang DQ and Britt DE: LYRIC/AEG-1 overexpression modulates BCCIPalpha protein levels in prostate tumor cells. Biochem Biophys Res Commun 371: 333-338, 2008.
7. Thirkettle HJ, Mills IG, Whitaker HC and Neal DE: Nuclear LYRIC/AEG-1 interacts with PLZF and relieves PLZF-mediated repression. Oncogene 28: 3663-3670, 2009.

8. Sarkar D, Park ES, Emdad L, Lee SG, Su ZZ and Fisher PB Molecular basis of nuclear factor-kappaB activation by astrocyte elevated gene-1. Cancer Res 68: 1478-1484, 2008.

9. Srivastava J, Robertson CL, Rajasekaran D, Gredler R, Siddiq A, Emdad L, Mukhopadhyay ND, Ghosh S, Hylemon PB, Gil G, et al: AEG-1 regulates retinoid $\mathrm{X}$ receptor and inhibits retinoid signaling. Cancer Res 74: 4364-4377, 2014.

10. Su P, Zhang Q and Yang Q: Immunohistochemical analysis of Metadherin in proliferative and cancerous breast tissue. Diagn Pathol 5: 38, 2010

11. Tokunaga E, Nakashima Y, Yamashita N, Hisamatsu Y, Okada S, Akiyoshi S, Aishima S, Kitao H, Morita M and Maehara Y: Overexpression of metadherin/MTDH is associated with an aggressive phenotype and a poor prognosis in invasive breast cancer. Breast Cancer 21: 341-349, 2014.

12. Xu C, Kong X, Wang H, Zhang N, Kong X, Ding X, Li X and Yang Q: MTDH mediates estrogen-independent growth and tamoxifen resistance by downregulating PTEN in MCF-7 breast cancer cells. Cell Physiol Biochem 33: 1557-1567, 2014.

13. Kavlashvili T, Jia Y, Dai D, Meng X, Thiel KW, Leslie KK and Yang S: Inverse relationship between progesterone receptor and Myc in endometrial cancer. PLoS One 11: e0148912, 2016.

14. Hanahan D and Weinberg RA: Hallmarks of cancer: The next generation. Cell 144: 646-674, 2011.

15. Hanahan D and Weinberg RA: The hallmarks of cancer. Cell 100: 57-70, 2000.

16. Gupta GP and Massagué J: Cancer metastasis: Building a framework. Cell 127: 679-695, 2006.

17. Roberts JM and Gammill H: Pre-eclampsia and cardiovascular disease in later life. Lancet 366: 961-962, 2005.

18. Mendelsohn ME and Karas RH: The protective effects of estrogen on the cardiovascular system. N Engl J Med 340: 1801-1811, 1999.

19. Gennari L, Merlotti D, De Paola V, Calabrò A, Becherini L, Martini G and Nuti R: Estrogen receptor gene polymorphisms and the genetics of osteoporosis: A HuGE review. Am J Epidemiol 161: 307-320, 2005 .

20. Saso S, Chatterjee J, Georgiou E, Ditri AM, Smith JR and Ghaem-Maghami S: Endometrial cancer. BMJ 343: d3954, 2011.

21. Khuda II II, Koide N, Noman AS, Dagvadorj J, Tumurkhuu G, Naiki Y, Komatsu T, Yoshida T and Yokochi T: Astrocyte elevated gene-1 (AEG-1) is induced by lipopolysaccharide as toll-like receptor 4 (TLR4) ligand and regulates TLR4 signalling. Immunology 128 (Suppl 1): e700-e706, 2009. 